\title{
Brain function rescue effect of lactate following hypoglycaemia is not an adaptation process in both normal and Type I diabetic subjects
}

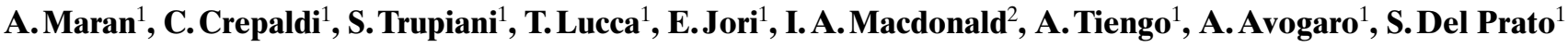 \\ ${ }^{1}$ Department of Clinical and Experimental Medicine, Division of Metabolic Diseases, University of Padua, Italy \\ ${ }^{2}$ School of Biomedical Sciences, University of Nottingham, UK
}

\section{Abstract}

Aims/hypothesis. We have previously shown that lactate protects brain function during insulin-induced hypoglycaemia. An adaptation process could, however, not be excluded because the blood lactate increase preceded hypoglycaemia.

Methods. We studied seven healthy volunteers and seven patients with Type I (insulin-dependent) diabetes mellitus with a hyperinsulinaemic $(1.5 \mathrm{mU}$. $\mathrm{kg}^{-1} \cdot \mathrm{min}^{-1}$ ) stepwise hypoglycaemic clamp (4.8 to $3.6,3.0$ and $2.8 \mathrm{mmo} / \mathrm{l})$ with and without Na-lactate infusion $\left(30 \mu \mathrm{mol} \cdot \mathrm{kg}^{-1} \cdot \mathrm{min}^{-1}\right)$ given after initiation of hypoglycaemic symptoms.

Results. The glucose threshold for epinephrine response was similar (control subjects $3.2 \pm 0.1$ vs $3.2 \pm$ 0.1 , diabetic patients $=3.5 \pm 0.1$ vs $3.5 \pm 0.1 \mathrm{mmol} / \mathrm{l}$ ) in both studies. The magnitude of the response was, however, blunted by lactate infusion (AUC; control subjects $65 \pm 28$ vs $314 \pm 55 \mathrm{nmol} / 1 / 180 \mathrm{~min}$, zenith $=2.6 \pm 0.5$ vs $4.8 \pm 0.7 \mathrm{nmol} / 1, p<0.05$; diabetic patients $=102 \pm 14$ vs $205 \pm 40 \mathrm{nmol} / 1 / 180 \mathrm{~min}$, zenith $=1.4 \pm 0.4$ vs $3.2 \pm 0.3 \mathrm{nmol} / 1, p<0.01)$. The glucose threshold for symptoms was also similar $(\mathrm{C}=\mathrm{au}-$ tonomic $3.0 \pm 0.1$ vs $3.0 \pm 0.1$, neuroglycopenic $=2.8 \pm 0.1$ vs $2.9 \pm 0.1 \mathrm{mmol} / \mathrm{l}, \quad \mathrm{D}=$ autonomic $3.2 \pm 0.1$ vs $3.2 \pm 0.1$, neuroglycopenic $3.1 \pm 0.1$ vs
$3.2 \pm 0.1 \mathrm{mmol} / \mathrm{l})$ but peak responses were significantly attenuated by lactate (score at $160 \mathrm{~min}$ $\mathrm{C}=2.6 \pm 1$ vs $8.8 \pm 1$, and $0.4 \pm 0.4$ vs $4.8 \pm 1$, respectively; $p=0.02-0.01, \mathrm{D}=1.3 \pm 0.5$ vs $6.3 \pm 1.7$, and $2.3 \pm 0.6$ vs $5.7 \pm 1.1 p=0.07-0.02)$. Cognitive function deteriorated in both studies at similar glucose thresholds $(C=3.1 \pm 0.1$ vs $3.0 \pm 0.1, D=3.2 \pm 0.1$ vs $3.3 \pm 0.2 \mathrm{mmol} / \mathrm{l})$. Although in normal subjects a much smaller impairment was observed with lactate infusion ( $\Delta$ four-choice reaction time at $160 \mathrm{~min}=$ $22 \pm 12$ vs $77 \pm 31 \mathrm{~ms} ; p=0.02$ ), in Type I diabetic patients lactate infusion was associated with an improvement in cognitive dysfunction $(0.2 \pm 0.4$ vs $-38 \pm 0.2 \Delta \mathrm{ms}, p=0.0001$ ).

Conclusion/interpretation. A blood lactate increase after the development of hypoglycaemic symptoms reduces counterregulatory and symptomatic responses to insulin-induced hypoglycaemia and favours brain function rescue both in normal and diabetic subjects. These findings confirm that lactate is an alternative substrate to glucose for cerebral metabolism under hypoglycaemic conditions. [Diabetologia (2000) 43: 733-741]

Keywords Hypoglycaemia, lactate, cognitive function, reaction time.
Received: 30 September 1999 and in revised form: 10 February 2000

Corresponding author: A. Maran, M.D., Cattedra di Malattie del Metabolismo, Via Giustiniani 2, I-35123 Padova, Italy
Patients with Type I (insulin-dependent) diabetes mellitus receiving intensified insulin therapy and having strict metabolic control are exposed to the risk of recurrent episodes of hypoglycaemia [1]. Defective counterregulatory and symptomatic responses to hypoglycaemia are responsible for reduced awareness of hypoglycaemia [2] which can aggravate cerebral dysfunction by increasing the occurrence and severity 
of hypoglycaemia in these patients [3], thus leading into a vicious cycle [4]. Although glucose represents the main fuel for the brain, it is also possible that under conditions of glucose shortage, brain function is maintained by the uptake of other energetic substrates.

We have shown that an alternative fuel for the brain, such as lactate, is able to diminish symptoms, counterregulatory responses and cognitive dysfunction during hypoglycaemia induced by insulin [5]. This result has been confirmed $[6,7]$ with similar results in diabetic patients [8].

In all these studies blood lactate concentration was, however, increased before the actual development of hypoglycaemic symptoms. Therefore, it is difficult to define whether the observed response was due to brain adaptation or a real shift from brain uptake of glucose to lactate under conditions of short-term glucose shortage. For lactate to play a physiological part, it must be shown that hypoglycaemic counterregulation is reduced by an increase in blood lactate concentration which occurs after hypoglycaemia has developed and counterregulation triggered.

Therefore, we designed this study to assess whether a physiological increase in blood lactate that follows the development of symptomatic hypoglycaemia is able to rescue brain function. Because the counterregulatory response could be different due to an adaptation process to more frequent blood glucose fluctuations both normal subjects and Type I diabetic patients were studied.

\section{Methods}

Subjects. Participants in the study were seven normal volunteers (six men and one woman) and seven Type I male diabetic patients. Their demographic features are summarised in Table 1. Patients regularly attended the Type I Diabetes Clinic of the University Hospital of Padova on a monthly basis. The incidence of severe hypoglycaemia, biochemical hypoglycaemia (by home blood glucose monitoring diaries), overall metabolic control $\left(\mathrm{HbA}_{1 \mathrm{c}}\right)$ and awareness of hypoglycaemia was available for 2 years for each patient. These patients were selected because they never presented a severe hypoglycaemic event (loss of consciousness, need for a third assistance or need for glucagon injection) during the 2 years nor was there anamnestic recording for such events. Normal volunteers had

Table 1. Demographic features of study subjects (median and range)

\begin{tabular}{lll}
\hline & Normal volunteers & Type I diabetic \\
\hline$n$ & 7 & 7 \\
$\mathrm{Sex}(\mathrm{M} / \mathrm{F})$ & $6 / 1$ & 7 \\
$\mathrm{Age}$ & $27(25-29)$ & $29(26-38)$ \\
$\mathrm{BMI}\left(\mathrm{kg} / \mathrm{m}^{2}\right)$ & $25(24-27)$ & $23.7(23.3-24.1)$ \\
$\mathrm{HbA}_{1 \mathrm{c}} \%$ & - & $8.3(7.2-10.3)$ \\
Duration of disease (years) & - & $16(12-24)$ \\
Insulin dose (U/day) & - & $51(41-56)$ \\
\hline
\end{tabular}

no hypertension, ischaemic heart disease or family history of diabetes. All subjects consumed an isocaloric diet, containing more than $200 \mathrm{~g}$ carbohydrate a day for at least 3 days before each study. All subjects were recruited on a consecutive basis. All investigations were carried out in the post-absorptive state, after a 12 to 14-h overnight fast. The protocol was reviewed and approved by the institutional review board. The purpose, nature, and potential risk of the study were explained to all subjects and informed written consent was obtained before their participation.

Experimental protocol. All subjects underwent two hypoglycaemic clamp studies with or without concomitant intravenous lactate infusion as described below. The two studies were carried out in a random order according to a computergenerated randomisation as single blind studies.

Diabetic patients were admitted to the metabolic ward of the University Hospital, Padova, the night before the study. Intermediate insulin was discontinued and plasma glucose was controlled throughout the night by intravenous insulin infusion $\left(0.1-0.3 \mathrm{mU} \cdot \mathrm{kg}^{-1} \cdot \mathrm{min}^{-1}\right)$ to ensure overnight normoglycaemia (4.0-6-0 mmol/l).

Normal volunteers were admitted in the morning of the study, after an overnight fast. Alcohol and cigarette smoking was forbidden during the preceding day. At 0800 hours, a 20gauge Teflon catheter was inserted into an antecubital vein for the infusion of all test substances. A second catheter was inserted retrogradely into a wrist vein of the ipsilateral arm for blood sampling, and the hand placed in a hot box $\left(55-60^{\circ} \mathrm{C}\right)$ to achieve arterialisation of venous blood [9]. Each subject was then trained on the measurement of the four-choice-reaction time test [10], doing at least five measurements until stable readings were achieved.

The experimental protocol consisted of two hyperinsulinaemic stepwise hypoglycaemic clamp studies done in a random order. In all subjects, after collection of three baseline blood samples, a primed and continuous $\left(1.5 \mathrm{mU} \cdot \mathrm{kg}^{-1} \cdot \mathrm{min}^{-1}\right)$ infusion of soluble insulin (Actrapid HM, Novo Nordisk, Denmark) was started. Plasma glucose was maintained at $4.8 \mathrm{mmol} / \mathrm{l}$ for $40 \mathrm{~min}$ according to a negative feedback principle by an appropriate glucose infusion (glucose $20 \% \mathrm{w} / \mathrm{v}$ ). It was then reduced by steps to 3.6, 3.0 and $2.8 \mathrm{mmol} / \mathrm{l}$. Each step lasted 40 min with the new plasma glucose goal being achieved in 10-20 min. After completion of the last hypoglycaemic step, plasma glucose was returned to $4.8 \mathrm{mmol} / 1$ for a final $20 \mathrm{~min}$.

To rule out an adaptation process due to early provision of alternative substrate, lactate infusion needed to be started after the triggering of the neuroglycopenic response. We therefore chose to start a sodium-lactate infusion $\left(30 \mu \mathrm{mol} \cdot \mathrm{kg}^{-1}\right.$. $\mathrm{min}^{-1}$ ) arbitrarily after recognition of the typical hypoglycaemic symptoms such as sweating and palpitations. In the repeat study, an equivalent volume of $0.9 \%$ sodium chloride solution was infused instead of lactate.

Blood samples were taken every 10-20 min for measurements of catecholamines, glucagon, insulin, C peptide, intermediary metabolites, free fatty acids and lactate. A symptom score was recorded every $20 \mathrm{~min}$ by means of a semi-quantitative questionnaire. Symptoms were assessed on a linear analogue scale, ranking a total of 40 different symptoms from 0 (absent) to 6 (very severe). Autonomic symptoms were calculated from the sum of the scores assigned to sweat, shakiness, anxiety, hotness or heart pounding. Neuroglycopenic scores were calculated as the sum of the scores attributed to dizziness, difficulty in concentrating, confusion, irritability or blurred vision [11].

After completion of each symptom questionnaire, a fourchoice-reaction time measurement was obtained. This test measures the time required for the subject to clear a symbol 

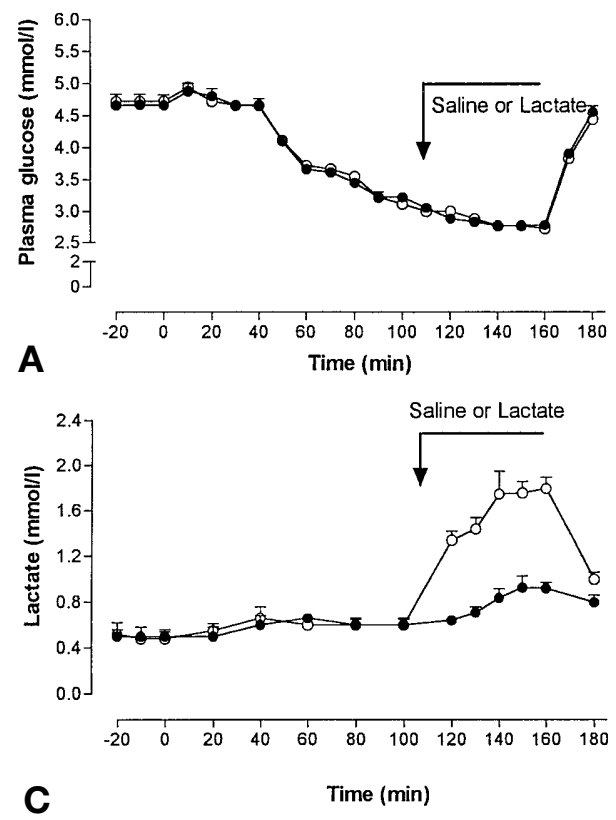

Fig. 1A-D. Plasma glucose concentrations and blood lactate concentrations (mean \pm SEM) during the hypoglycaemic clamp studies with Na-lactate $(O)$ or saline $(\mathbf{O})$ infusion in normal volunteers $(\mathbf{A}, \mathbf{C})$ and diabetic patients $(\mathbf{B}, \mathbf{D})$. Infusions were started after the development of symptoms for hypoglycaemia

appearing at random in one of four quadrants on a computer screen, by pressing a key in a corresponding position on a keypad. The results represent the average time used for a total of 500 tests over $5 \mathrm{~min}$. As shown previously with repeated measures over a 220-min euglycaemic insulin clamp study, the coefficient of variation is $1 \%$ for accuracy of the test and $2 \%$ for speed [10].

Biochemical assays. Plasma glucose concentration during the clamp studies was measured at the bedside with a glucose oxidase method (Yellow Springs Instrument Company, Yellow Springs, Ohio, USA). Plasma catecholamine concentrations were determined by high-performance liquid chromatography [12] and plasma concentrations of free insulin and $\mathrm{C}$ peptide were measured by standard radioimmunoassay procedures [13]. A fluorimetric method was used to measure blood lactate, intermediary metabolites [14], and free fatty acids [15].

Calculations and statistical analysis. A significant hormone response was defined as an increase of at least two standard deviations above mean basal (euglycaemic) levels on at least two consecutive measurements. An increase of two points or more of the score for autonomic and neuroglycopenic symptoms was considered to be of significant importance. Glucose threshold for each response was defined as the plasma glucose concentration associated with the first significant change as defined above.

Glucose thresholds, peaks of responses and the incremental areas under the curve of plasma hormone responses in the presence or absence of lactate were compared by paired $t$ test analysis or Wilcoxon signed rank test when data were not normally distributed. All data are expressed as means \pm SEM. A $p$ value less than 0.05 was considered significant.
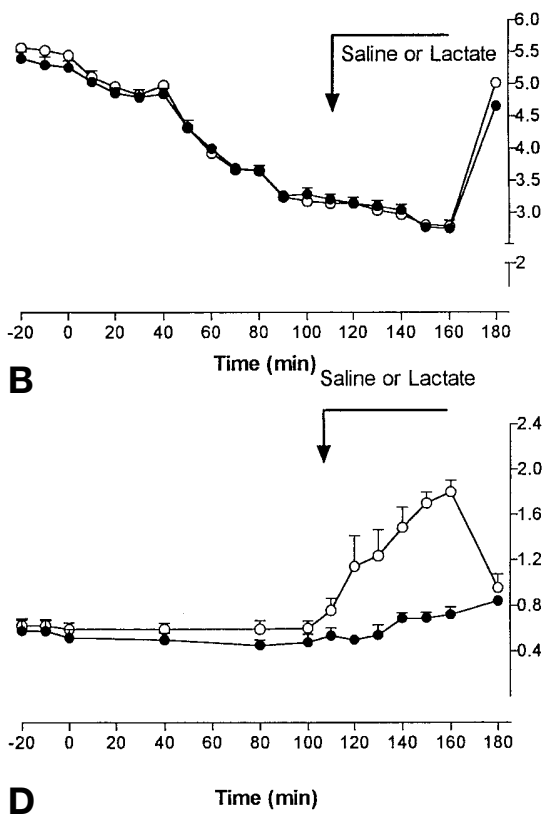

\section{Results}

Plasma glucose, insulin, C-peptide and lactate concentrations (Fig. 1). The metabolic and hormonal conditions preceding the two tests (with and without $\mathrm{Na}-$ lactate infusion) were similar in control subjects and Type I diabetic patients. There were no differences in the baseline plasma concentrations of substrates and hormones between control subjects and diabetic patients with the exception of higher plasma insulin and lower plasma C-peptide concentrations in the latter. After insulin infusion, plasma insulin concentration was similar in both groups in both hypoglycaemic hyperinsulinaemic clamp studies (control subjects: $119 \pm 12$ vs $119 \pm 6 \mu \mathrm{U} / \mathrm{ml}$; diabetic patients: $105 \pm 6$ vs $105 \pm 7 \mu \mathrm{U} / \mathrm{ml})$.

Plasma glucose profiles during the two stepwise hypoglycaemic clamp studies were superimposable (Fig. 1), with an identical rate of decline (control subjects: $0.01 \pm 0.0001$ vs $0.01 \pm 0.0001$; diabetic subjects: $0.01 \pm 0.0001$ vs $0.01 \pm 0.0001 \mathrm{mmol} / 1 / \mathrm{min})$, plasma glucose nadir (Controls: $2.8 \pm 0.04$ vs $2.8 \pm 0.05$; diabetic subjects: $2.8 \pm 0.03$ vs $2.8 \pm 0.06 \mathrm{mmol} / \mathrm{l})$. With saline infusion, blood lactate concentration increased to $0.9 \pm 0.1 \mathrm{mmol} / \mathrm{l}$ in normal control subjects and to $0.7 \pm 0.1$ at $160 \mathrm{~min}$ (both $p<0.03$ ) in diabetic patients. Lactate infusion increased blood lactate concentration (control subjects: $1.8 \pm 0.1$; diabetic patients: $1.75 \pm 0.2 \mathrm{mmol} / \mathrm{l}$ at $160 \mathrm{~min}$; both $p<0.001 \mathrm{vs}$ baseline; Fig.1) compared with the control hypoglycaemic visit. The starting time for infusions was $108 \pm 6 \mathrm{~min}$ and $107 \pm 8 \mathrm{~min}$ for lactate and saline solutions, respectively, in normal subjects whereas the infusions began later in diabetic patients $(115 \pm 5 \mathrm{~min}$ and $116 \pm 4 \mathrm{~min}$, respectively). 

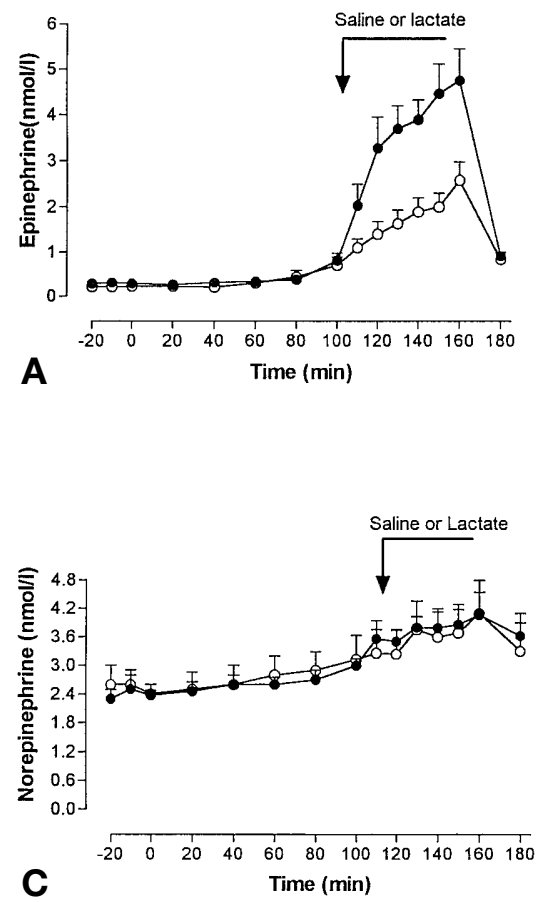

Fig.2 A-D. Plasma catecholamine concentrations during the hypoglycaemic clamp studies with Na-lactate $(\bigcirc)$ or saline infusion $(\mathbf{O})$ in normal volunteers $(\mathbf{A}, \mathbf{C})$ and diabetic patients $(\mathbf{B}, \mathbf{D})$

Plasma counterregulatory hormones. Basal plasma concentrations of counterregulatory hormones were similar in both studies both in diabetic patients and normal control subjects. In the control study (saline infusion), counterregulatory hormones increased in response to hypoglycaemia with no significant difference between normal and diabetic subjects. The plasma glucose thresholds for epinephrine and norepinephrine responses were $3.2 \pm 0.1$ and $3.1 \pm$ $0.1 \mathrm{mmol} / 1$ in normal volunteers, $3.5 \pm 0.1$ and $3.2 \pm 0.1 \mathrm{mmol} / \mathrm{l}$ in the diabetic patients, respectively $(p=0.8)$. In the repeat study, the plasma glucose threshold was identified at the same level (control
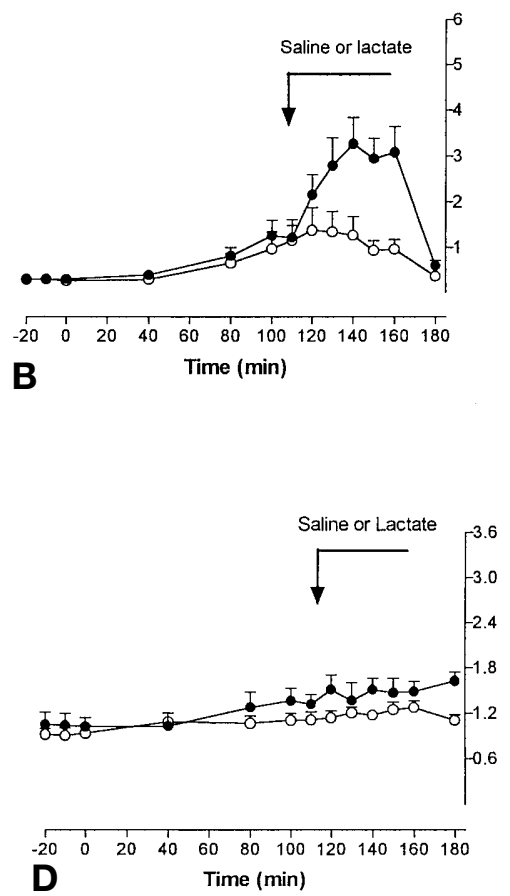

subjects: $3.2 \pm 0.1$ and $3.1 \pm 0.2 \mathrm{mmol} / \mathrm{l}$; diabetic patients: $3.5 \pm 0.1$ and $3.2 \pm 0.1 \mathrm{mmol} / \mathrm{l}$ ).

In the control study, the peak plasma catecholamine concentrations during hypoglycaemia were lower in diabetic patients than in control subjects (epinephrine $4.8 \pm 0.7$ vs $3.2 \pm 0.3 \mathrm{nmol} / 1, p=0.04$; norepinephrine $4.1 \pm 0.5$ vs $1.5 \pm 0.15, p=0.02$; Fig. 2 ). In both groups, the infusion of Na-lactate after detection of hypoglycaemic symptoms was associated with lower peak plasma epinephrine concentrations (peak $=2.6 \pm 0.5$ and $1.4 \pm 0.4 \mathrm{nmol} / \mathrm{l} ; p=0.001$, in control subjects and diabetic patients, respectively, both $p<0.005-0.001$ vs saline). Lactate infusion did not, however, affect the plasma norepinephrine concentrations $($ peak $=4.13 \pm 0.7$ and $1.3 \pm 0.1 \mathrm{nmol} / \mathrm{l}$, respectively). Similarly, the incremental area under the curve was much lower with lactate, than saline infusion, for plasma epinephrine (control subjects $=165 \pm 28$ vs $314 \pm 55 \mathrm{nmol} / \mathrm{l} / 180 \mathrm{~min}, p=0.01$; diabetic patients $=205 \pm 40$ vs $102 \pm 14 \mathrm{nmol} / \mathrm{l} /$

Table 2. Pre-test plasma concentration of hormones and substrates

\begin{tabular}{|c|c|c|c|c|}
\hline & \multicolumn{2}{|c|}{ Normal subjects } & \multicolumn{2}{|c|}{ Type I diabetic subjects } \\
\hline & Saline & Lactate & Saline & Lactate \\
\hline Plasma glucose $(\mathrm{mmol} / \mathrm{l}$ & $4.7 \pm 0.2$ & $4.8 \pm 0.1$ & $5.4 \pm 0.05$ & $5.2 \pm 0.1$ \\
\hline Plasma C peptide (nmol/l) & $1.2 \pm 0.1$ & $1.15 \pm 0.1$ & $0.1 \pm 0.01$ & $0.1 \pm 0.01$ \\
\hline Blood lactate $(\mathrm{mmol} / \mathrm{l})$ & $0.81 \pm 0.02$ & $0.88 \pm 0.1$ & $0.57 \pm 0.1$ & $0.65 \pm 0.1$ \\
\hline Plasma NEFA (mmol/l) & $0.47 \pm 0.1$ & $0.43 \pm 0.07$ & $0.1 \pm 0.02$ & $0.1 \pm 0.01$ \\
\hline Plasma glucagon (pmol/l) & $72.5 \pm 8$ & $73 \pm 11$ & $85 \pm 9$ & $88 \pm 11$ \\
\hline Plasma growth hormone $(\mu \mathrm{g} / \mathrm{l})$ & $1.8 \pm 0.7$ & $2 \pm 1$ & $1 \pm 0.6$ & $1 \pm 0.4$ \\
\hline Plasma cortisol (nmol/1) & $220 \pm 26$ & $226 \pm 14$ & $347 \pm 30$ & $324 \pm 59$ \\
\hline
\end{tabular}



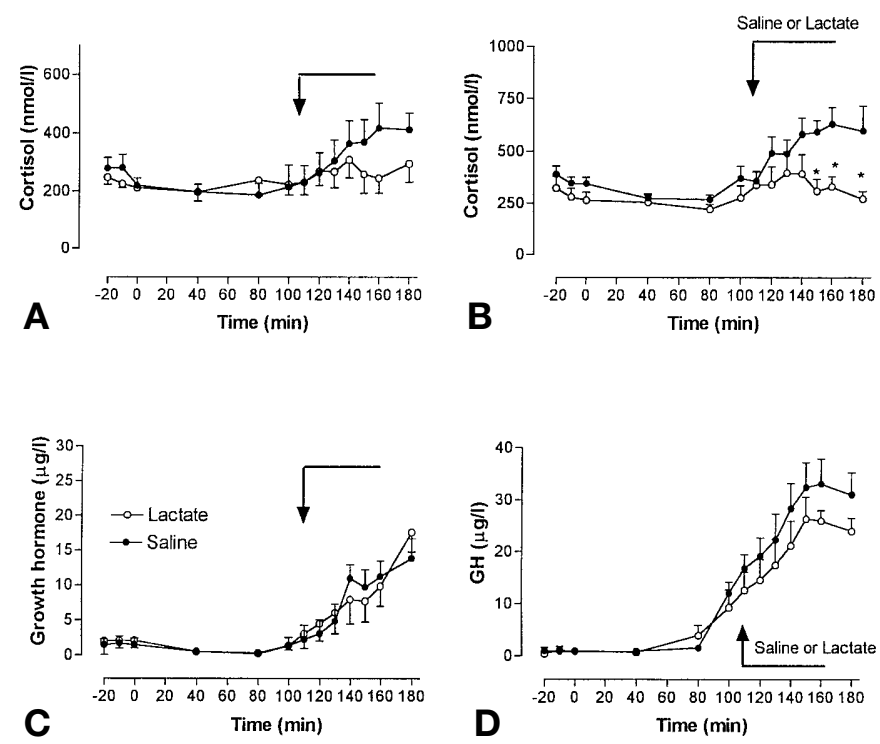

Fig.3 A-D. Plasma growth hormone and cortisol concentrations during hypoglycaemic clamp studies with Na-lactate $(\bigcirc)$ or saline $(\mathbf{O})$ infusion in normal volunteers $(\mathbf{A}, \mathbf{C})$ and diabetic ptients $(\mathbf{B}, \mathbf{D}) .{ }^{*} p<0.05$

$180 \min , p=0.03$ ) but not for plasma norepinephrine (control subjects $=544 \pm 125$ vs $500 \pm 75 \mathrm{nmol} / \mathrm{l} /$ $180 \mathrm{~min}$; diabetic patients $=206 \pm 34$ vs $183 \pm 17$ $\mathrm{nmol} / \mathrm{l} / 180 \mathrm{~min}$ ).

Plasma cortisol concentration increased in response to hypoglycaemia in both control subjects and diabetic patients. In both groups lactate infusion was associated with a blunted cortisol response compared with the saline study. (Fig. 3)

In normal volunteers, lactate treatment did not affect the glucagon response to hypoglycaemia (glucose threshold $=3.1 \pm 0.1$ vs $3.2 \pm 0.1 \mathrm{mmol} / 1, \quad p=0.45$; AUC $=126.2 \pm 17.6$ vs $147.1 \pm 12.7 \quad \mathrm{nmol} / \mathrm{ml} /$ $180 \mathrm{~min}$ ). In diabetic patients, the glucagon response to hypoglycaemia could not be detected and there was no effect of lactate infusion (Fig. 4). The growth hormone response was also not affected by lactate treatment after hypoglycaemic symptoms developed

Fig. 4A, B. Plasma glucagon responses during hypoglycaemic clamp studies in normal volunteers $(\mathbf{A})$ and diabetic patients (B)
(Fig.3). Nevertheless, diabetic patients showed a prompter and more pronounced response than control subjects.

Symptomatic responses (Fig. 5). Both control subjects and diabetic patients experienced hypoglycaemic symptoms at similar plasma glucose concentrations with no change due to Na-lactate infusion (control subjects $=3.0 \pm 0.1$ vs $3.0 \pm 0.1$ and $2.8 \pm 0.1$ vs $2.9 \pm 0.1 \mathrm{mmol} / \mathrm{l} ;$ diabetic patients $=3.2 \pm 0.1$ vs $3.2 \pm 0.1$ and $3.1 \pm 0.1$ vs $3.2 \pm 0.1 \mathrm{mmol} / \mathrm{l}$ for autonomic and neuroglycopenic symptoms, respectively). During the control study, hypoglycaemia was associated with a progressive worsening of the symptomatic reaction and this process was reduced by Na-lactate infusion. At $160 \mathrm{~min}$, in control subjects, the score was $2.6 \pm 0.1$ compared with $8.8 \pm 0.1$ and $0.4 \pm 0.4$ compared with $4.8 \pm 0.1$ for autonomic and neuroglycopenic symptoms, respectively $(p=0.01-0.02)$. In Type I diabetic patients the scores were $1.3 \pm 0.5$ compared with $6.3 \pm 1.7$ and $2.3 \pm 0.6$ compared with $5.7 \pm 1.1(p=0.02-0.07)$ (Fig. 5).

Cognitive function. Treatment with Na-lactate did not affect the glucose threshold for cognitive dysfunction in either control subjects $(3.1 \pm 0.1 \mathrm{vs} 3.0 \pm 0.1 \mathrm{mmol} /$ 1) or Type I diabetic patients $(3.3 \pm 0.2$ vs $3.2 \pm 0.1 \mathrm{mmol} / \mathrm{l}$ for diabetic patients). In normal control subjects lactate infusion prevented, however, further deterioration in the progression of hypoglycaemia (Fig. 6). Thus, at 160 min the change in fourchoice-reaction time was lower with lactate infusion (22 \pm 12 vs $77 \pm 31 \mathrm{~ms} ; p=0.02)$.

In Type I diabetic patients progression of hypoglycaemia did not worsen cognitive function to a greater extent. Nevertheless, lactate infusion was associated with improved cognitive function to the point that at 160 min a complete recovery in cognitive dysfunction was apparent (Fig. 6).

\section{Discussion}

As previously shown, counterregulation can be altered, compared with normal people, in long-standing Type I diabetic patients. Nonetheless, in both normal and Type I diabetic people a rapid increase of

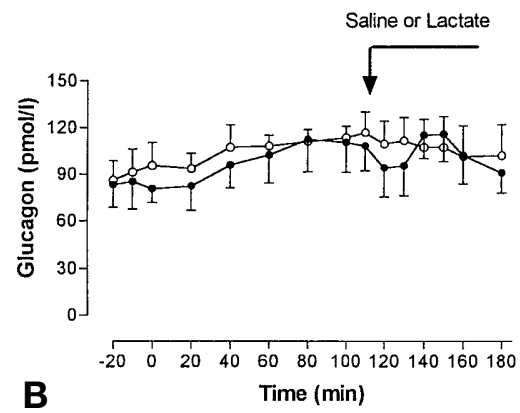



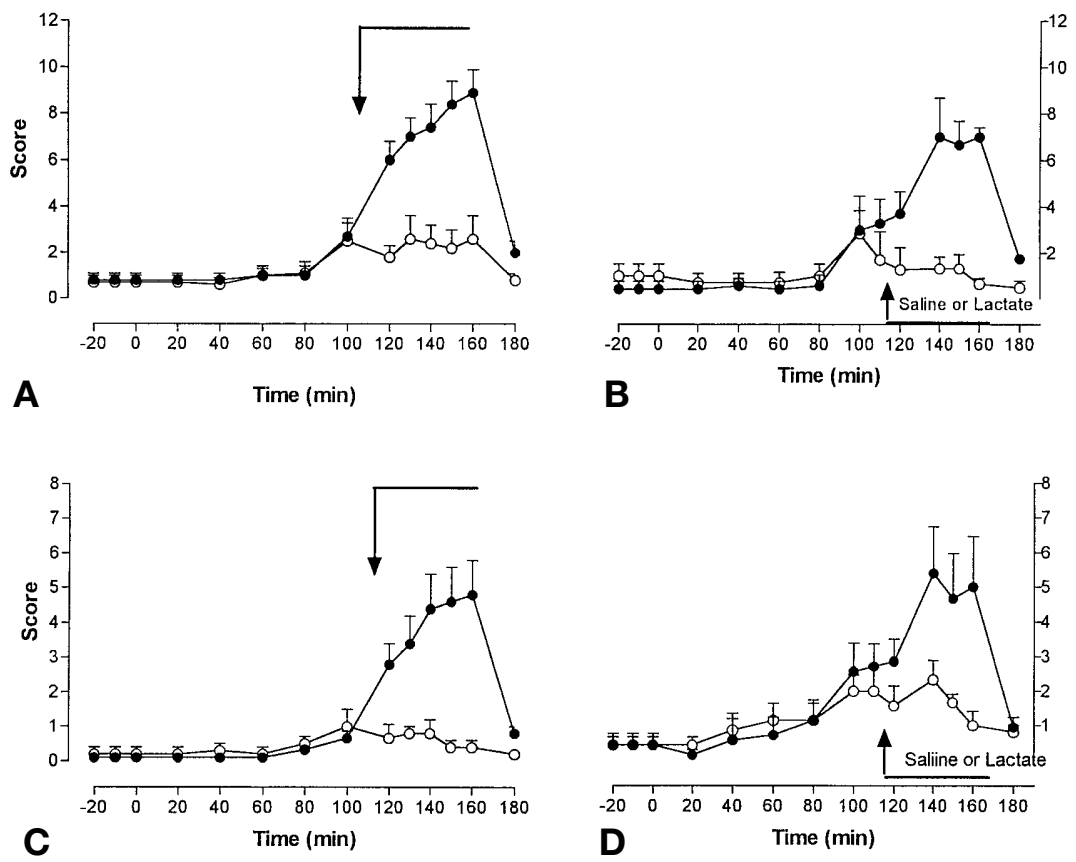

Fig.5 A-D. Symptoms scores during the hypoglycaemic clamp studies with Na-lactate $(\bigcirc)$ or saline infusion $(\mathbf{O})$ in normal volunteers $(\mathbf{A}, \mathbf{C})$ and diabetic patients $(\mathbf{B}, \mathbf{D})$. $\mathbf{A}$ and $\mathbf{B}$ show autonomic symptoms, and $\mathbf{C}$ and $\mathbf{D}$ show neuroglycopenic symptoms

blood lactate concentration after the development of hypoglycaemic symptoms is associated with a statistically significant reduction in plasma epinephrine response and prevention of cognitive function deterioration despite progressive reduction in plasma glucose concentrations. These effects of post-hypoglycaemic hyperlactacidaemia are consistent with the hypothesis that the brain rapidly shifts from glucose to lactate consumption when there is a glucose shortage and that this feature is preserved in Type I

Fig. 6A, B. Speed on the four-choice reaction time test during hypoglycaemia (ms) and delta difference at time $160 \mathrm{~min}$ during the hypoglycaemic clamp studies with Na-lactate $(\bigcirc)$ or saline infusion (O) in normal volunteers $(\mathbf{A})$ and diabetic patients $(\mathbf{B}) .(* p=0.02)$

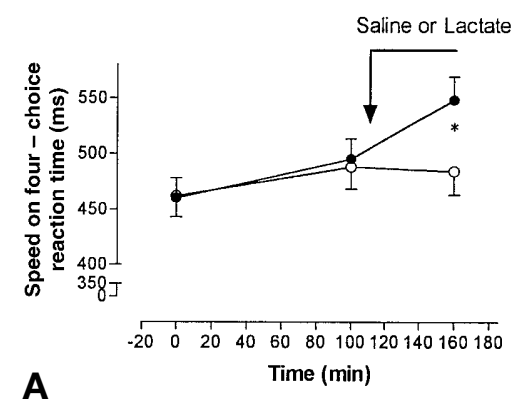

diabetic patients. This effect does not require adaptation in the brain sensor responsible for the initiation of protective responses to hypoglycaemia.

The idea that alternative fuels might provide energy to the central nervous system stems from the observation that during a prolonged fast, the human brain switches from glucose to ketones as a main energetic substrate [16]. Later on, it was shown that ketone body infusion diminishes the threshold and the magnitude of counterregulatory hormonal response to acute hypoglycaemia [17]. The physiological role of ketone bodies under conditions of insulin-induced hypoglycaemia is however, open to question. In diabetic patients, hypoglycaemia is mainly associated with absolute hyperinsulinaemia which causes inhibition of ketogenesis and low blood ketone concentrations. In contrast, blood lactate concentration tends to be high in insulin-treated diabetic patients [18], and becomes even higher under conditions of relative hyperinsulinaemia as it occurs, for instance, in the situation of increased physical activity [19] and inadequate insulin dose adjustment.

Lactate, therefore, is an ideal candidate for an alternative brain fuel under conditions of low ambient plasma glucose. This issue has been addressed previ-

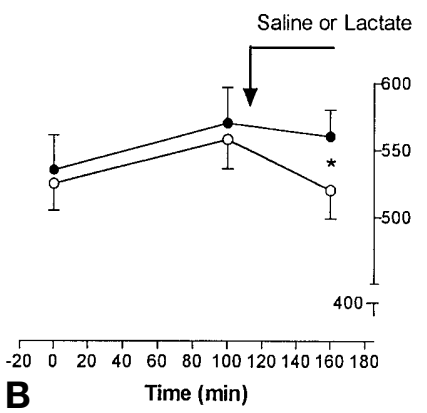


ously by us and others [5, 6], the results of those studies supporting a role for lactate as an alternative fuel for the brain under the condition of insulin-induced hypoglycaemia. The present study provides evidence that the complex mechanism responsible for the hormonal responses which might prevent severe neuroglycopenia can be rapidly reversed, even after its activation, by a non-glucose fuel such as lactate. In the previous studies, indeed, lactate infusion preceded hypoglycaemia. In our previous study, for instance, lactate infusion was begun $60 \mathrm{~min}$ before induction of hypoglycaemia, whereas in the study by the other group [6] there was at least a 30-min gap between the beginning of the lactate treatment and the reduction of plasma glucose below $4.0 \mathrm{mmol} / \mathrm{l}$. Under these conditions, an adaptation process cannot be ruled out to account for the increase observed in the glycaemic threshold, reduced magnitude of autonomic and neuroglycopenic symptoms, counterregulation hormone responses and cognitive dysfunction. To gain direct evidence for lactate as a true alternative fuel for brain energy metabolism, we have chosen to infuse lactate only after the hypoglycaemic symptoms had developed, that is after the counterregulatory response has been fully triggered in both normal subjects and Type I diabetic patients.

Alkalosis in itself does not account for the Na-lactate effect on glucose counterregulation and cognitive dysfunction [5]. Thus, we conclude that lactate must be avidly taken up by the brain areas responsible for sensing the lack of availability of glucose as a fuel for energy metabolism and for the activation of counterregulatory hormone response. This conclusion is supported by several experimental data. In animals, lactate uptake by the brain increases during hypoglycaemia [20]. In hypoglycaemic dogs, lactate can contribute up to $25 \%$ of the brain energy requirement [21]. Moreover, local ventromedial hypothalamic glucopenia triggers the counterregulatory responses to hypoglycaemia in rats [22], whereas this response is blunted in animals with selective lesions of the same nuclei. Finally, selective lactate perfusion of this structure suppresses counterregulation in rats with systemic hypoglycaemia. The concomitant reduction of hormonal response and prevention of cognitive dysfunction with an increase of blood lactate concentration after development of hypoglycaemia observed in our study suggests that lactate is used as a metabolic fuel in the cerebral cortex as well as in the hypothalamus.

From a qualitative point of view, results of this study resemble those observed with lactate infusion preceding insulin-induced hypoglycaemia $[5,6]$ with the main effect exerted on plasma epinephrine concentrations. No effect was detected on plasma glucagon concentration and only a limited increase in the plasma norepinephrine concentrations occurred. We have no explanation for the lack of statistically signif- icant response of glucagon, a finding which confirms both our previous studies [5, 7]. Glucagon secretion is, however, believed to be under peripheral rather than central control [23]. Alternatively, it might be speculated that the peripheral glucose sensors can metabolise lactate when there is a glucose shortage. Finally, glucagon secretion could be prevented by concomitant constant increase of the plasma insulin concentration as a consequence of the hyperinsulinaemic clamp. It is also notable that the same behaviour occurred in both normal subjects and Type I diabetic patients. Thus, the lack of glucagon response in diabetic people cannot be the simple consequence of diabetes duration [24].

The limitation to our findings might be seen in an apparently trivial physiological impact of a condition characterised by increased plasma insulin, low plasma glucose and a concomitant increase in blood lactate concentrations. Nevertheless, physiological and pathological conditions exist where a role for lactate as an alternative fuel for the hypoglycaemic brain can be shown. Physiological conditions characterised by glucose shortage and lactate abundance, such as strenuous exercise, are not associated with symptomatic hypoglycaemia [25]. Under these conditions, when plasma glucose concentration drops to values as low as $2.5 \mathrm{mmol} / \mathrm{l}$ or lower and blood lactate concentrations increase threefold, no symptoms are generally detected. Children affected by von Gierke's disease have a striking tolerance to hypoglycaemia. In this condition the deficiency of glucose-6-phosphatase causes hypoglycaemia and concomitant acceleration of hepatic glycolysis with enhanced lactate production. Tolerance to hypoglycaemia in these children is directly related to prevalent blood lactate concentration [26].

Another possible limitation of the study could be the blood lactate concentration attained as they might not be frequently encountered during the daily life of Type I diabetic patients. A more physiologic blood lactate concentration was, however, used than in previous studies. For instance in another study [6] plasma lactate concentration was as high as $5 \mathrm{mmol} / \mathrm{l}$ but in our study it reached a maximum concentration of $1.6 \mathrm{mmol} / \mathrm{l}$ in control subjects and $1.8 \mathrm{mmol} / 1$ in diabetic patients, values similar to those reported in diabetic patients after physical exercise [19]. More importantly, even if the blood lactate concentration attained in our study exceeded those found in normal daily life, our results are still of relevance for brain fuel metabolism and brain preservation in response to hypoglycaemia and support the hypothesis that lactate can be an important alternative fuel for the hypoglycaemic brain, or at least for some region of it. In our study, the four-reaction time test was used to assess cognitive function, which might explore specific cerebral pathways [27]. This possibility is supported by other results [28] showing that increasing 
plasma NEFA could reduce the catecholamine response and symptoms to hypoglycaemia without supporting cognitive function. The regional brain protection could also account for the main effect on adrenaline but not on the glucagon response observed in this and previous studies $[5,7]$.

If lactate can be seen as a rescue fuel in subjects experiencing acute hypoglycaemia, it could be argued that long-term exposure to mildly increased blood lactate concentration, as can occur in insulin-treated diabetic patients, could increase the threshold for glucose counterregulation and, thus, contribute for the hypoglycaemic unawareness of long-standing Type I diabetes. This hypothesis does not seem to be supported by our own data. The present study shows that rapid increase in lactate concentrations reverses cognitive dysfunction due to hypoglycaemia protecting to a similar extent both in normal subjects and Type I diabetic patients. Hypoglycaemia unawareness is characterised by a general defect in counterregulatory hormone secretion [29], whereas lactate provision mainly reduces the epinephrine response to hypoglycaemia. Moreover, although in normal subjects lactate prevented a further deterioration of cognitive function during sustained hypoglycaemia, cognitive function actually returned to normal in diabetic patients. The hypothesis that lactate can therefore cause a sort of "maladaptation" of the brain is not supported by our study. We studied Type I diabetic patients with moderate glycaemic control because of a relatively better preservation of counterregulatory responses and normal glucose thresholds for hypoglycaemic symptoms, therefore providing better chances to explore the effect of alternate substrate fuel on the hypoglycaemic brain. Whether there is a similar effect in patients with strict glycaemic control is not known.

The effect of the increase of blood lactate concentration on the epinephrine response, together with the major improvement in cognitive function suggest that when there is a glucose shortage, neuronal cells can rapidly switch to a different substrate. Nevertheless, it seems difficult at present to suggest an increase in blood lactate concentrations as a therapeutic approach to hypoglycaemia in Type I diabetic patients. It is not known, for instance, whether these results apply to Type I diabetic patients with asymptomatic hypoglycaemia or different degrees of glycaemic control.

An increase in blood lactate that follows the development of hypoglycaemia is capable of reducing the epinephrine response, as well as autonomic and neuroglycopenic symptom responses, to hypoglycaemia induced by insulin in both normal and Type I diabetic subjects. In both groups, lactate provision improves the cognitive function during hypoglycaemia. These effects occur rapidly; as such this does not represent an adaptation process. Therefore, increase in blood lactate that ensues after hypoglycaemia could be part of the redundant defensive mechanisms against insulin-induced hypoglycaemia, both in normal subjects and Type I diabetic patients.

Acknowledgements. This study was supported in part by the Juvenile Diabetes Foundation.

\section{References}

1. The Diabetes and Complication Trial Research Group (1993) The effect of intensive treatment of diabetes on the development and progression of long-term complications in insulin-dependent diabetes mellitus. N Engl J Med 329: 977-986

2. Amiel SA, Tamborlane WV, Simonson DC, Sherwin RS (1987) Defective glucose counterregulation after strict glycemic control of insulin-dependent diabetes mellitus. $\mathrm{N}$ Engl J Med 316: 1376-1383

3. Ryder RE, Owens DR, Hayes TM, Ghatei MA, Bloom SR (1990) Unawareness of hypoglycaemia and inadequate hypoglycaemic counterregulation:no causal relation with diabetic autonomic neuropathy. BMJ 301: 783-787

4. Clarke WL, Gonder-Frederick LA, Richards FE, Cryer PE (1991) Multifactorial origin of hypoglycemic symptom unawareness in IDDM. Association with defective glucose counterregulation and better glycemic control. Diabetes 40: 680-685

5. Maran A, Cranston I, Lomas J, Macdonald I, Amiel SA (1994) Protection by lactate of cerebral function during hypoglycaemia. Lancet 343: 16-20

6. Veneman T, Mitrakou A, Mokan M, Cryer P, Gerich J (1994) Effect of hyperketonemia and hyperlacticacidemia on symptoms, cognitive dysfunction, and counterregulatory hormone responses during hypoglycemia in normal humans. Diabetes 43: 1311-1317

7. King P, Parkin H, Macdonald IA, Barber C, Tattersall RB (1997) The effect of intravenous lactate on cerebral function during hypoglycaemia. Diabet Med 14: 19-28

8. King P, Kong MF, Parkin H, Macdonald IA, Barber C, Tattersall RB (1998) Intravenous lactate prevents cerebral dysfunction during hypoglycaemia in insulin-dependent diabetes mellitus. Clin Sci (Colch) 94: 157-163

9. Liu D, Moberg E, Kollind M, Lins PE, Adamson U, Macdonald IA (1992) Arterial, arterialized venous, venous and capillary blood glucose measurements in normal man during hyperinsulinaemic euglycaemia and hypoglycaemia. Diabetologia 35: 287-290

10. Maran A, Lomas J, Macdonald IA, Amiel SA (1995) Lack of preservation of higher brain function during hypoglycaemia in patients with intensively-treated IDDM. Diabetologia 38: 1412-1418

11. Hepburn DA, Deary IJ, Frier BM, Patrick AW, Quinn JD, Fisher BM (1991) Symptoms of acute insulin-induced hypoglycemia in humans with and without IDDM. Factoranalysis approach. Diabetes Care 14: 949-957

12. Macdonald IA, Lake DM (1985) An improved method for extracting catecholamines from body fluid. J Neurosci Methods 13: 239-248

13. Kuzuya H, Blix PM, Horwitz DL, Steiner DF, Rubenstein AH (1977) Determination of free and total insulin and Cpeptide in insulin- treated diabetics. Diabetes 26: 22-29

14. Amiel SA, Pottinger RC, Archibald HR et al. (1991) Effect of antecedent glucose control on cerebral function during hypoglycemia. Diabetes Care 14: 109-118 
15. De Feo P, Perriello G, De Cosmo S et al. (1986) Comparison of glucose counterregulation during short-term and prolonged hypoglycemia in normal humans. Diabetes 35 : 563-569

16. Owen OE, Morgan AP, Kemp HG, Sullivan JM, Herrara HG, Cahill GF (1967) Brain metabolism during fasting. J Clin Invest 46: 1590-1595

17. Amiel SA, Archibald HR, Chusney G, Williams AJ, Gale EA (1991) Ketone infusion lowers hormonal responses to hypoglycaemia:evidence for acute cerebral utilization of a non-glucose fuel. Clin Sci (Colch) 81: 189-194

18. Trevisan R, Nosadini R, Avogaro A et al. (1986) Type I diabetes is characterized by insulin resistance not only with regard to glucose, but also to lipid and amino acid metabolism. J Clin Endocrinol Metab 62: 1155-1162

19. Berger M, Berchtold P, Cuppers HJ et al. (1977) Metabolic and hormonal effects of muscular exercise in juvenile type diabetics. Diabetologia 13: 355-365

20. Avogaro A, Nosadini R, Doria A et al. (1990) Substrate availability other than glucose in the brain during euglycemia and insulin-induced hypoglycemia in dogs. Metabolism 39: $46-50$

21. Nemoto EM, Hoff JT, Severinghaus JW (1974) Lactate uptake and metabolism by brain during hyperlactatemia and hypoglycemia. Stroke 5: 48-53

22. Borg MA, Sherwin RS, Borg WP, Tamborlane WV, Shulman GI (1997) Local ventromedial hypothalamus glucose perfusion blocks counterregulation during systemic hypoglycemia in awake rats. J Clin Invest 99: 361-365

23. Wise JE, Hendler R, Felig P (1973) Evaluation of alpha cell function by infusion of alanine in normal and diabetic subjects. N Engl J Med 288: 487-490

24. Bolli G, De Feo P, Perriello G et al. (1984) Mechanisms of glucagon secretion during insulin-induced hypoglycemia in man. Role of the beta cell and arterial hyperinsulinemia. J Clin Invest 73: 917-922

25. Miller JM, Coyle EF, Sherman WM et al. (1983) Effect of glycerol feeding on endurance and metabolism during prolonged exercise in man. Med Sci Sports Exerc 15: 237-242

26. Fernandes J, Berger R, Smit GP (1984) Lactate as a cerebral metabolic fuel for glucose-6-phosphatase deficient children. Pediatr Res 18: 335-339

27. Amiel SA (1998) Cognitive function testing in studies of acute hypoglycaemia:rights and wrongs? Diabetologia 41: 713-719

28. Evans ML, Matyka K, Lomas J et al. (1998) Reduced counterregulation during hypoglycemia with raised circulating nonglucose lipid substrates: evidence for regional differences in metabolic capacity in the human brain? J Clin Endocrinol Metab 83: 2952-2959

29. Mokan M, Mitrakou A, Veneman T et al. (1994) Hypoglycemia unawareness in IDDM. Diabetes Care 17: 1397-1403 